\title{
Pain and accuracy of focal laser treatment for diabetic macular edema using a retinal navigated laser (Navilas ${ }^{\circledR}$ )
}

This article was published in the following Dove Press journal:

Clinical Ophthalmology

25 February 2012

Number of times this article has been viewed

\author{
Marcus Kernt* \\ Raoul E Cheuteu* \\ Sarah Cserhati \\ Florian Seidensticker \\ Raffael G Liegl \\ Julian Lang \\ Christos Haritoglou \\ Anselm Kampik \\ Michael W Ulbig \\ Aljoscha S Neubauer \\ Department of Ophthalmology, \\ Ludwig Maximilian University \\ of Munich, Germany \\ *These authors contributed equally to \\ this study
}

Correspondence: Marcus Kernt Department of Ophthalmology, Ludwig Maximilian University of Munich, Mathildenstr 8, 80336 Muenchen, Germany $\mathrm{Tel}+498951603811$

Fax +498951605160

Email marcus.kernt@med.uni-muenchen.de
Aim: To investigate treatment-related pain and the accuracy of navigated laser photocoagulation in the treatment of clinically significant macular edema.

Methods: Focal laser treatment of diabetic macular edema in 54 consecutive patients was digitally planned on fundus images and performed using the navigated laser photocoagulation system Navilas $^{\circledR}$ (OD-OS GmbH, Teltow, Germany). Treatment-related pain was quantified on a visual analog scale directly after treatment and compared with a matched control group who received conventional laser treatment $(n=46)$. In addition, for Navilas-treated patients, the accuracy of spot placement on color images was analyzed 1 month after treatment.

Results: In total, 5423 laser spots (mean 100 per eye) were analyzed. With navigated treatment, $90 \%$ of laser spots were visible on color images, of which $96 \%$ were within $100 \mu \mathrm{m}$ from the target. Eighty percent of the laser spots were placed and visible within the $100 \mu \mathrm{m}$ target on an intention-to-treat basis for color imaging. Optical coherence topography confirmed that laser effects were limited to the outer retina. Treatment-related pain following navigated laser photocoagulation was significantly lower than that of conventional laser treatment (1.6 vs 4.4 on a visual analog scale, $P<0.001)$.

Conclusion: Navigated laser effects could be visualized to a high percentage on post-treatment color images, and their location showed a high concordance to targeted areas. Patients reported that treatment-related pain following Navilas laser photocoagulation was significantly lower than pain following conventional laser treatment.

Keywords: diabetic retinopathy, navigated laser therapy, pattern laser, diabetes

\section{Introduction}

Diabetic retinopathy (DR) is the most frequent cause of new cases of blindness among adults of working age. ${ }^{1}$ Diabetic retinopathy can cause vision loss through several mechanisms, with diabetic macular edema (DME) being a major cause..$^{2,3}$

Laser photocoagulation has played a central role in the treatment of both DR and DME in the past 30 years. The Diabetic Retinopathy Study (DRS) in 1971 marked the progression of photocoagulation clinical trials, which involved more than 1700 patients enrolled across 15 medical centers. A significant result of the DRS and other population-based studies (such as the Early Treatment of Diabetic Retinopathy Study) was the demonstration that retinal laser photocoagulation was a safe and effective treatment for both DR and DME. Since then, laser photocoagulation has become a well-established standard therapy for DME, and it has recently been supplemented by pharmacological interventions, particularly anti-vascular endothelial growth factor treatment. ${ }^{4}$ 
Meyer-Schwickerath introduced retinal photocoagulation therapy in the 1950s using the xenon arc photocoagulator, and Campbell et al first described successful retinal laser photocoagulation in 1964 - a tremendous evolution of retinal laser therapy has taken place since this time. ${ }^{5,6}$ Most importantly, we have seen photocoagulation become a less painful and more accurate treatment of retinal diseases, and several technical advances have been introduced to laser therapy including subthreshold techniques ${ }^{7}$ and pattern laser generation. ${ }^{8}$ However, the advantages of computerized precision ("eye tracking") known from, eg, refractive surgery, have not become available despite several technical approaches towards that goal. ${ }^{9}$ Of note, in DME a protocol targeting microaneurysms appears to be advantageous versus a non-targeted early treatment diabetic retinopathy study (ETDRS) laser protocol, ${ }^{10}$ which may hint the importance of targeting precision when performing laser treatments. In addition, essential diagnostics for the planning of laser treatment, such as fluorescein angiography, were mostly separated from the actual treatment device until the development of the navigated laser photocoagulation system Navilas ${ }^{\circledR}$ (OD-OS $\mathrm{GmbH}$, Teltow, Germany).

Navilas is a novel fundus imaging and laser treatment device that allows imaging (infrared, color, and fluorescein angiography) and integrated laser treatment of the retina. It includes pattern laser generation and a laser planning feature. ${ }^{11,12}$ Besides documentation, safety, and patient comfort, the main theoretical advantages of Navilas use lie in retina navigation, which may help to improve the clinical outcomes of laser treatment. In focal laser treatment, Navilas offers computerized image and target assistance systems, resulting in high precision and a theoretical reproducibility of $60-110 \mu \mathrm{m} .{ }^{13}$ The integration of diagnostic information into the device may help to overcome shortcomings during the process of transferring laser surgery protocols and potential loss of accuracy, helping retinal physicians to achieve better outcomes when treating their patients. An increase in patient comfort and reduction of treatment-related pain may also result from this novel technology.

We therefore investigated clinical in vivo accuracy and treatment-related pain in patients undergoing focal laser treatment with the Navilas system for diabetic macular edema.

\section{Methods}

\section{Navilas}

The Navilas device and its operation have been described elsewhere. ${ }^{11}$ In brief, Navilas combines imaging, laser application planning, and laser treatment in a single computer-controlled device. It fundamentally differs from most other laser devices by not being added to a slit lamp but is instead a scanning slit-based instrument: it uses approximately 25 images per second in imaging or treatment mode. For focal laser treatment, the field of view is $50^{\circ}$, and this is displayed on a monitor. The optical resolution of the instrument used in this study was $1280 \times 1024$ pixels resulting in 20-26 pixels/degree. Due to the slit-imaging principle, color images are obtained with high contrast and sharpness. ${ }^{13}$ Another difference to slit lamp-based laser devices is the touch-screen monitor used for imaging, planning, and treating fundus changes. This allows the retina specialist to plan laser spots on the screen for focal treatments, and then apply semi-automated patterns and single spots as appropriate. The pre-planned targets are stabilized on the fundus and a pre-positioning mode is provided by the system that automatically advances the aiming beam from each pre-planned target to the next. For treatment, the surgeon actuates the laser manually after he or she has verified the target lock.

\section{Patients treated with navigated laser photocoagulation}

Consecutive patients were recruited from the outpatient clinic of the Department of Ophthalmology, Ludwig Maximilian University of Munich, Germany. Patients were included if they had diabetes (based on WHO criteria) and were eligible for focal laser treatment as defined by the ETDRS criteria. Written informed consent was obtained from all patients. The device was a fully CE-approved laser at the time of this study. The study conformed to the principles expressed in the Declaration of Helsinki, and approval was obtained from the Ludwig Maximilian University's Institutional Review Board. Patients were excluded if they suffered from eye diseases involving the posterior pole other than diabetic retinopathy (such as age-related macular degeneration), but they were not excluded for media opacities.

\section{Imaging}

Navilas imaging was performed with fully dilated pupils after the clinical examination and before laser therapy, and consisted of taking and saving several color images. Color imaging with Navilas was repeated 1 month after laser treatment and compared with pre-treatment images. Assessment of treatment success was performed by one experienced grader. Additionally, spectral domain optical coherence topography (SD-OCT) was performed 1 month after laser treatment (Heidelberg Engineering, Heidelberg, Germany). 


\section{Laser planning and treatment}

Laser planning and treatment was performed according to the ETDRS principles, not specifically targeting microaneurysms. A color image was obtained with the Navilas device and used to plan laser spot treatments by applying automated patterns and single spots as appropriate on the color image, thus generating a detailed treatment plan (Figure 1). It took less than 5 minutes to plan the treatment for each eye.

For laser application, a navigated, semi-automatic pattern laser application was conducted based on the treatment plan. Treatment spot size was always set at $100 \mu \mathrm{m}$, and the treatment time per spot was 100 milliseconds. The power settings of the green $(532 \mathrm{~nm})$ frequency doubled neodymium-doped yttrium aluminium garnet (Nd:YAG) laser were adjusted manually from a standard $100 \mathrm{~mW}$ so that spots showed a moderate whitening. The pre-positioning mode was used to automatically advance the targeting aiming beam from each targeted retinal position to the next position after the aiming beam and pre-planned target were stabilized on the living fundus. The surgeon actuated the laser manually after verifying the target lock. An alternating infrared to color video fundus visualization mode was applied to allow posttreatment observation of the retinal burn for 2-3 seconds after each application without patient discomfort.

\section{Grading of images}

The laser spots visible on the Navilas color images 1 month after treatment were compared to the laser treatment plan by overlaying the laser treatment plan with the post-treatment color image (Figure 2). The resulting image was post-processed using ImageJ (National Institutes of Health, Bethesda, MD) to improve the visibility of laser spots: the contrast was enhanced, the background reduced, and the image was zoomed to the region of interest. The post-processed images (Figure 2) were used to determine whether each treatment spot touched or overlapped the targeted $100 \mu \mathrm{m}$ laser spot (this was given a binary result: "match" or "no match"). The SD-OCT images were investigated for changes in the inner retinal layers overlying the laser spots. All data were collected in a Microsoft Excel 2000 spreadsheet (Microsoft Corporation, Redmond, WA) and analyzed using SPSS software (v 17.0 for Windows; IBM Corp, Armonk, NY).

\section{Evaluation of treatment-related pain after Navilas treatment}

Immediately after treatment, patients were asked to quantify their pain on a visual analog scale (VAS; $0=$ no pain; $10=$ very bad pain). To evaluate treatment-related pain after Navilas treatment versus conventional treatment, data were

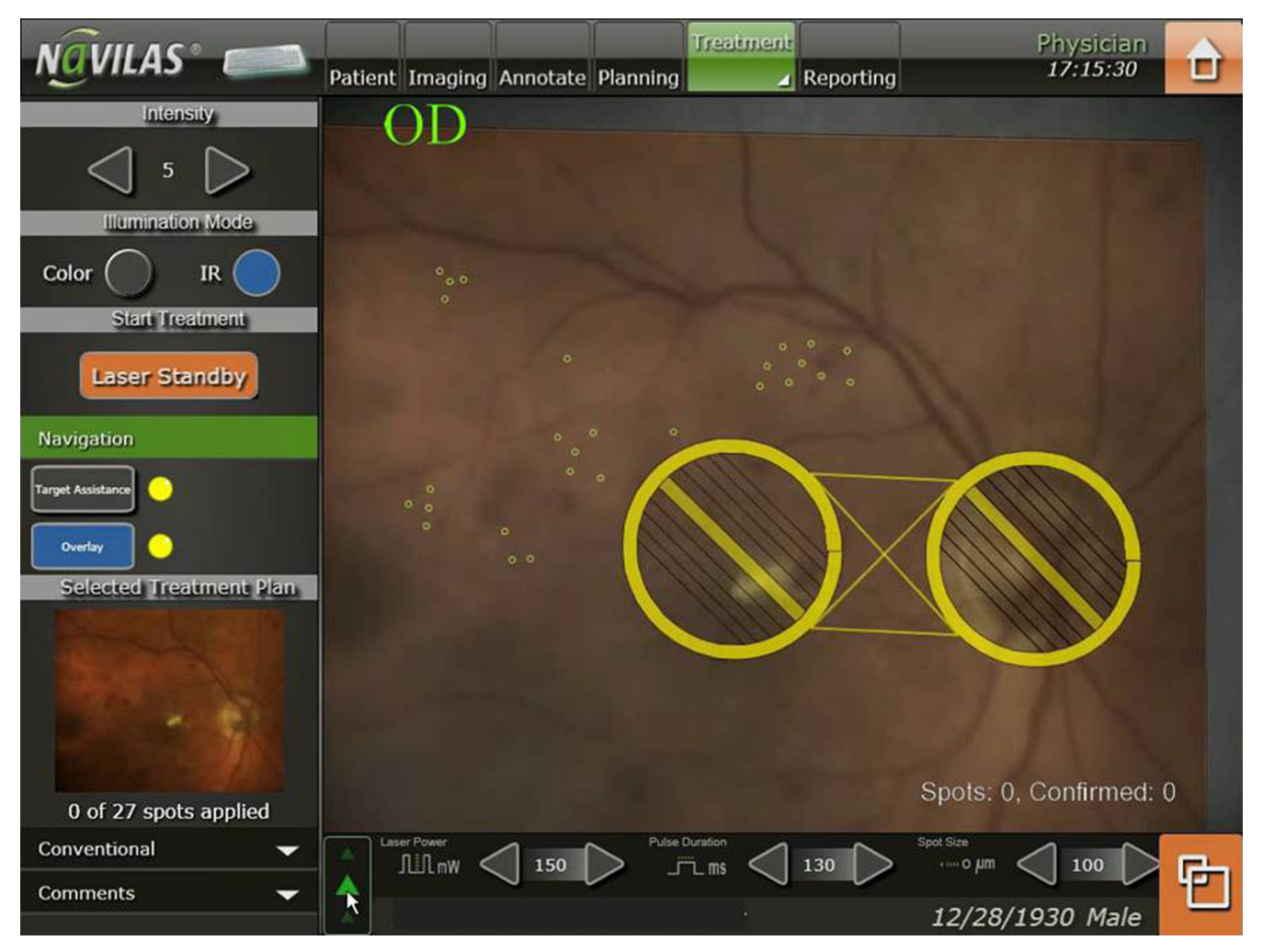

Figure I Pre-planned focal laser spots (small circles) in the Navilas ${ }^{\circledR}$ system on color image. The large circles indicate non-treatment zones. 


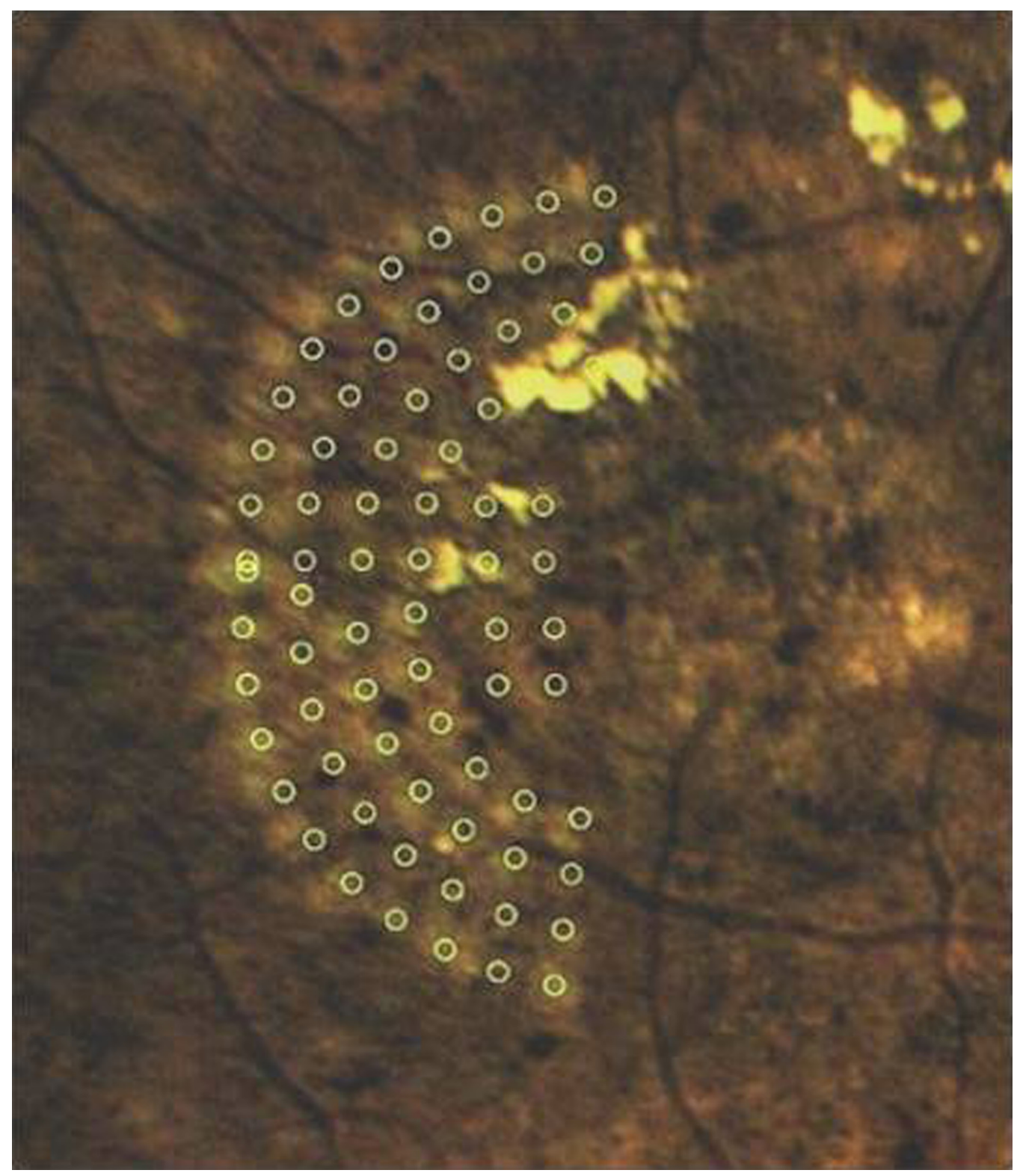

Figure 2 Pre-planned focal laser spots (circles) overlaid with post-processed color image I month after laser treatment with Navilas ${ }^{\circledR}$.

collected from the 54 patients treated with the Navilas system as well as from a matched control group of 46 subjects treated with a conventional laser (532 $\mathrm{nm}$ wave-length, VISULAS 532s, Carl Zeiss Meditec AG, Jena, Germany; laser settings: $100 \mathrm{~mW}$ power, 100 millisecond pulse duration, $100 \mu \mathrm{m}$ spot size). All patients were treated for DME at the authors' diabetes clinic during the same period. The two groups were analyzed and compared using SPSS software. For statistical testing, $P<0.05$ was considered significant.

\section{Results}

In the Navilas group, mean $\mathrm{HbA} 1 \mathrm{C}$ was $7.8 \%$ ( $\pm 4.2 \mathrm{SD})$, while in the matched control group that received conventional laser treatment the mean $\mathrm{HbA1C}$ was 7.4 ( $\pm 3.8 \mathrm{SD})$. Mean central retinal thickness (CRT) before treatment was $402 \mu \mathrm{m}$ $( \pm 154 \mu \mathrm{m}$ SD) in the Navilas group and $388 \mu \mathrm{m}( \pm 172 \mu \mathrm{m}$ $\mathrm{SD})$ in the control group.

\section{Treatment-related pain after Navilas treatment}

Patients who received Navilas treatment quantified posttreatment pain on the VAS with a mean of $1.6(\mathrm{SD}=1.0)$, significantly lower $(P<0.001)$ than for the control group which had a mean of $4.4(\mathrm{SD}=1.8)$ (Figure 3$)$. Significantly fewer (mean $43 \pm 36, P<0.001$ ) laser spots were applied to patients in the conventional laser group than those in the Navilas group. 


\section{Quantification of treatment-related pain}

No pain Very bad pain
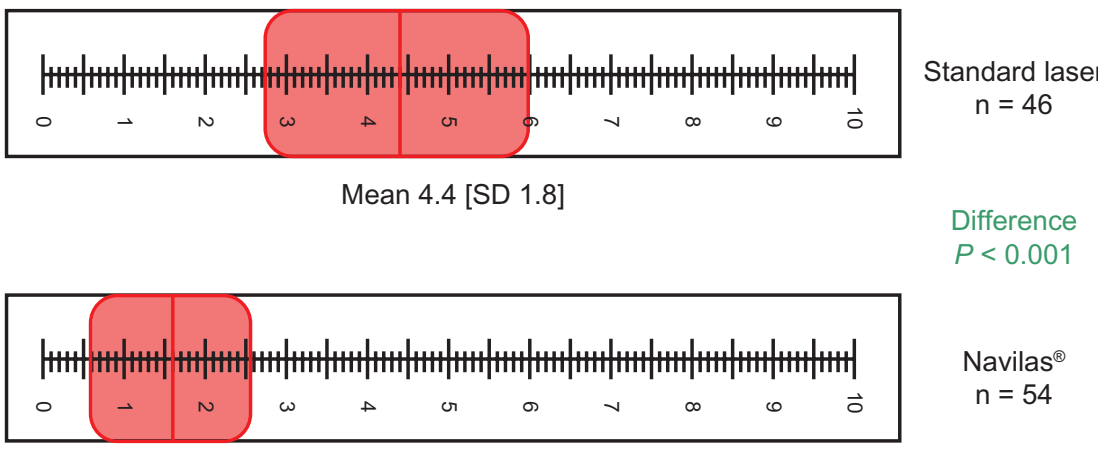

Navilas ${ }^{\circledR}$

$\mathrm{n}=54$

Mean 1.6 [SD 1.0]

Figure 3 Patient's treatment-related pain after navigated focal laser therapy with Navilas ${ }^{\circledR}$ was quantified by using a VAS.

Abbreviations: SD, standard deviation; VAS, visual analog scale.

\section{Accuracy of navigated retinal laser photocoagulation with Navilas}

A mean of $100 \pm 86$ spots per eye were pre-planned for treatment in 54 patients. All patients had clinically significant macular edema (as defined by the ETDRS) caused by type 2 diabetes. In total, 25 right and 29 left eyes were treated. The mean patient age was $61 \pm 12$ years. In total, 5423 pre-planned laser spots were applied and analyzed, and no complications occurred. Of those laser spots, 4888 (90\%) were visible in the 1 month color images (analysis per-protocol, PP). Mean hit-rate of visible spots was $96 \%$. On an intention-to-treat (ITT) analysis based on color image burn evaluation the hit-rate was $80 \%$ (Figure 4). Retinal OCT morphology of laser spots confirmed that laser lesions were limited to the outer retina, particularly the retinal pigment epithelium and photoreceptor layer (Figure 5).

\section{Discussion}

The ETDRS demonstrated the effectiveness of focal photocoagulation in eyes with macular edema. ${ }^{2}$ In macular laser photocoagulation (focal or modified grid), the main goal is to solve leakage arising from macular vessels by photocoagulating photoreceptors. This results in a reduced need for oxygen in the outer retina, allowing oxygen to diffuse from the choroid to the inner retina, so reducing hypoxia. ${ }^{14}$ In addition, increased oxygen tension in the inner retina helps to decrease tissue edema and improve vision by autoregulatory vasoconstriction and reduced hydrostatic pressure in the capillaries and venules. ${ }^{14,15}$ Therefore, focal photocoagulation, recently supplemented by treatments such as vascular endothelial growth factor treatment, is still the standard treatment. Nevertheless, in combined treatment, the use of modified laser protocols seems to be advantageous, ${ }^{4}$ and these data underscore the importance of precision laser targeting. Moreover, inadequate application of macular laser photocoagulation may be associated with loss of vision, diminished visual field, increased scotoma, reduced color vision, and reduced contrast sensitivity. ${ }^{16,17}$

The present study investigated the clinical accuracy of focal retinal laser application using a navigated, semiautomatic pattern laser. By utilizing computerized laser surgeon support, a $96 \%$ hit rate was achieved on an analyzable ("per-protocol") basis and $80 \%$ on an ITT basis. A target size of $100 \mu \mathrm{m}$ spots was applied to all patients, demonstrating the device's high laser spot application accuracy. The study does have some shortcomings such as missing clinical outcome data, a lack of follow-up, and a limited number of patients. It is also noted that the computerized laser surgeon support systems such as Navilas's pre-positioning mode, target stabilization, and target assistance require complex and fast real-time image processing, which may result in errors. Although those occurred rarely in our patient series, the final actuating of the laser needs to be performed by the surgeon. In addition, it is possible to continue with manual treatment as a fallback in case the electronic systems do not operate adequately.

The strengths of the system include image quality and remarkable laser spot accuracy on an ITT basis, especially given the limited evaluation properties of color images per se. Still, the present study's results demonstrate the high accuracy of the Navilas system. The authors believe that the current study demonstrates for the first time that 


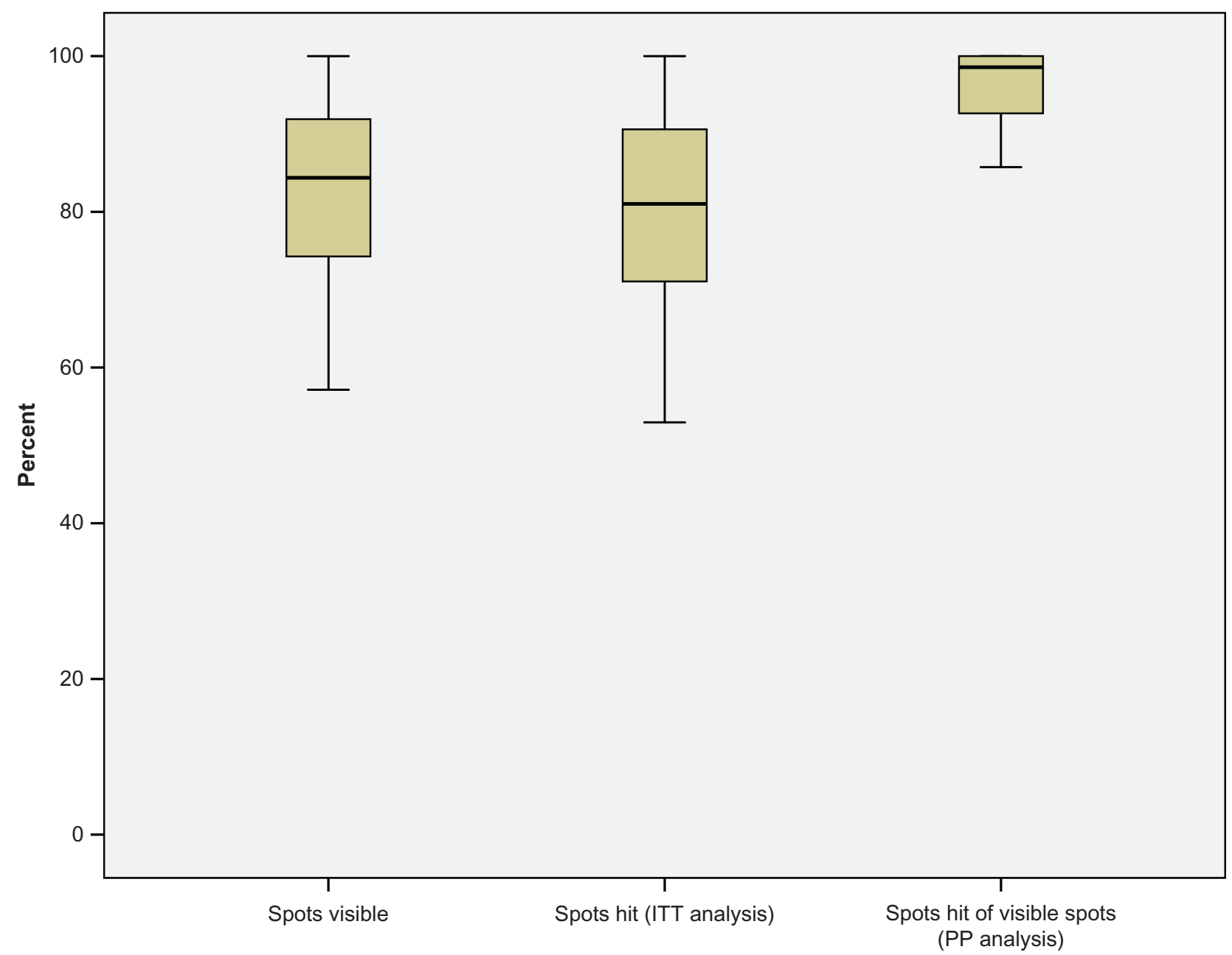

Figure 4 Accuracy of in vivo Navilas ${ }^{\circledR}$ treatment based on $n=54$ and $100 \pm 86$ spots per patient. In total, 5423 pre-planned laser spots were applied and analyzed. Of those laser spots, 4888 (90\%) were visible in the I month color images. The mean hit-rate of visible spots (color imaging) was $80 \%$ on an intention-to-treat (ITT) basis and $96 \%$ on a per-protocol (PP) basis.

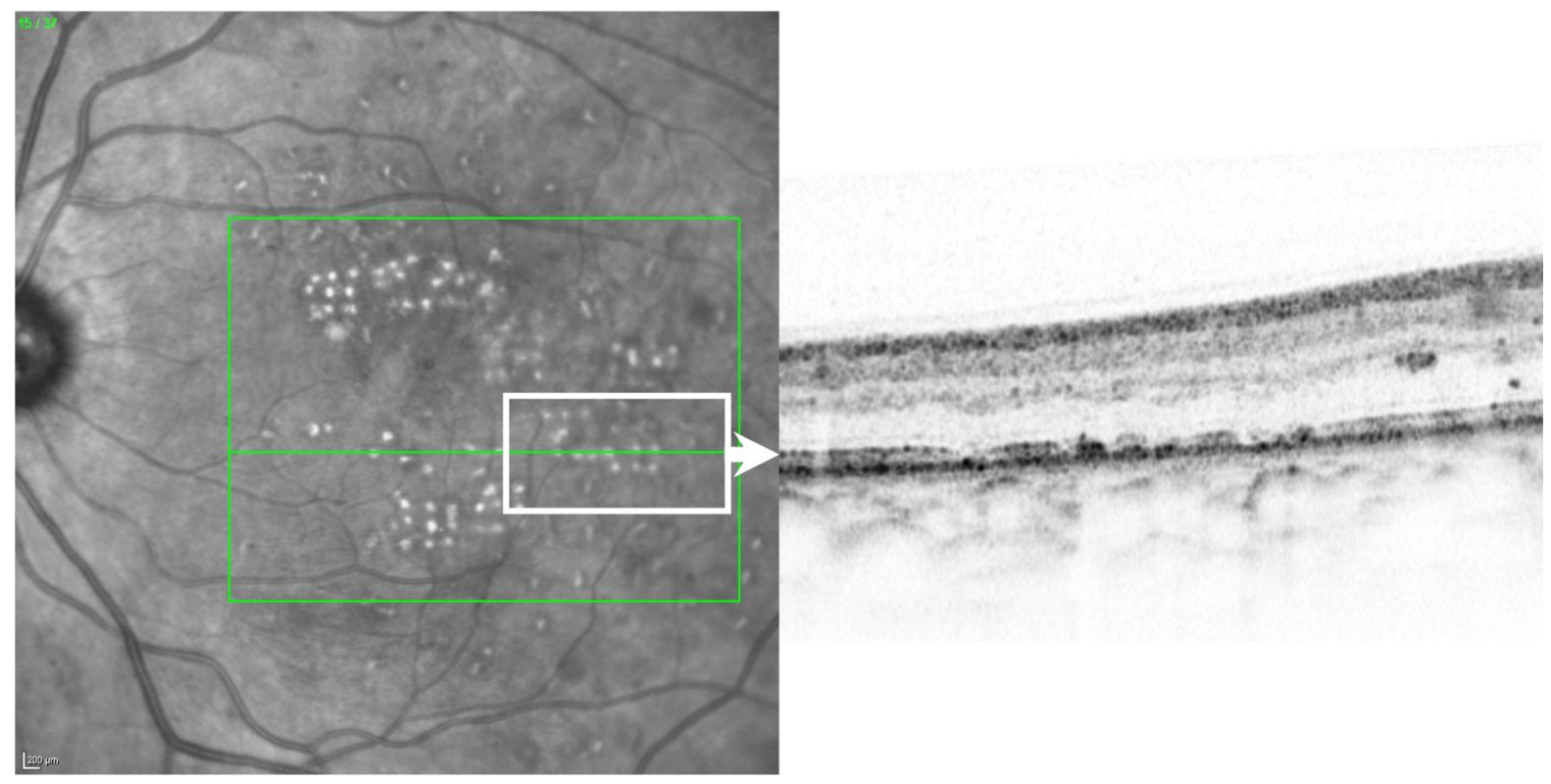

Figure 5 Example of the retinal optical coherence topography morphology of laser spots. 
$100 \mu \mathrm{m}$ pre-defined targets can be hit in vivo with very high precision by a navigated laser system. In an independent study, Kozac et al showed similar results targeting retinal microaneurysms with the device. ${ }^{18}$ The clinical value of targeting micronaneurysms in DME is disputed, ${ }^{10}$ but this technique may provide data on accuracy as they are anatomical landmarks on the fundus. In contrast to the work by Freeman's group we measured the device's ability to hit predefined fundus spots, which demonstrates accuracy with a given size target. While this approach has higher image processing requirements, it can be more universally applied to other diseases than DME, where improvements in accuracy may also have value, such as in venous occlusions or age-related macular degeneration. Navilas may serve as a platform for the development of new technology and its application in the treatment of those diseases or in special laser applications, such as subthreshold technology.

Patients suffering from DME do not always respond sufficiently to focal laser photocoagulation, which may be because laser treatment cannot always meet the patient's medical requirements. There are many reasons for this, but it appears to be quite common for patients not to accept or understand the need for laser treatment, and many report that treatment is uncomfortable or painful. The tracking of pain has been found to be a helpful diagnostic tool when evaluating the patient's treatment-related pain. ${ }^{19,20}$ By using a VAS the patient bypasses the cognitive level of the brain, resulting in a truer representation of pain. ${ }^{19,20}$

In our study, patients were asked to quantify treatmentrelated pain by using a VAS, and those who received navigated focal laser photocoagulation rated this as significantly less painful than conventional slit lamp-based laser photocoagulation (1.6 vs 4.4 respectively on a scale from 1 [no pain] to 10 [very strong pain]). This implicates another potential advantage of navigated laser therapy with Navilas, and the use of devices such as this may result in better patient compliance and acceptance of this treatment option.

In summary, this paper presents data that demonstrates that the Navilas system is accurate when targeting predefined targets in vivo in DME focal laser therapy. This not only suggests that such a computer-supported retinal navigation focal laser therapy is feasible but also demonstrates an accuracy of $80 \%$ on an intention-to-treat basis and $96 \%$ on a per-protocol basis. In addition, patients reported significantly less treatment-related pain following navigated focal laser photocoagulation compared to patients treated with a conventional laser. This may result in a higher acceptance of macular laser therapy in DME patients, which may help to improve treatment outcomes.

\section{Acknowledgments}

The authors thank Mrs S Guthmann and Mrs N Ehsani for expert technical assistance with OCT imaging.

\section{Disclosure}

Marcus Kernt and Aljoscha S Neubauer are consultants for OD-OS GmbH.

\section{References}

1. Icks A, Trautner C, Haastert B, Berger M, Giani G. Blindness due to diabetes: population-based age- and sex-specific incidence rates. Diabet Med. 1997;14(7):571-575.

2. Photocoagulation for diabetic macular edema. Early Treatment Diabetic Retinopathy Study report number 1. Early Treatment Diabetic Retinopathy Study research group. Arch Ophthalmol. 1985;103(12):1796-1806.

3. Early photocoagulation for diabetic retinopathy. ETDRS report number 9. Early Treatment Diabetic Retinopathy Study Research Group. Ophthalmology. 1991;98(5 Suppl):766-785.

4. Soheilian M, Ramezani A, Obudi A, et al. Randomized trial of intravitreal bevacizumab alone or combined with triamcinolone versus macular photocoagulation in diabetic macular edema. Ophthalmology. 2009;116(6):1142-1150.

5. Campbell CJ, Noyori KS, Rittler MC, Innis RE, Koester CJ. The application of fiber laser techniques to retinal surgery. Arch Ophthalmol. 1964;72:850-857.

6. Meyer-Schwickerath G. Light coagulation; a method for treatment and prevention of the retinal detachment. Albrecht Von Graefes Arch Ophthalmol. 1954;156(1):2-34. German.

7. Nakamura Y, Mitamura Y, Ogata K, Arai M, Takatsuna Y, Yamamoto S. Functional and morphological changes of macula after subthreshold micropulse diode laser photocoagulation for diabetic macular oedema. Eye (Lond). 2010;24(5):784-788.

8. Bolz M, Kriechbaum K, Simader C, et al. In vivo retinal morphology after grid laser treatment in diabetic macular edema. Ophthalmology. 2010;117(3):538-544.

9. Naess E, Molvik T, Ludwig D, et al. Computer-assisted laser photocoagulation of the retina - a hybrid tracking approach. J Biomed Opt. 2002;7(2):179-189

10. Fong DS, Strauber SF, Aiello LP, et al. Comparison of the modified Early Treatment Diabetic Retinopathy Study and mild macular grid laser photocoagulation strategies for diabetic macular edema. Arch Ophthalmol. 2007;125(4):469-480.

11. Kernt M, Cheuteu R, Vounotrypidis E, et al. Focal and panretinal photocoagulation with a navigated laser $\left(\mathrm{NAVILAS}^{\circledR}\right)$. Acta Ophthalmol. 2011;89(9):e662-e664.

12. Kozak I, Kim JS, Oster SF, Chhablani J, Freeman WR. Focal navigated laser photocoagulation in retinovascular disease: clinical results in initial case series. Retina. 2011. [Epub ahead of print.]

13. Kernt M, Neubauer AS, Liegl R, et al. Cytoprotective effects of a blue light-filtering intraocular lens on human retinal pigment epithelium by reducing phototoxic effects on vascular endothelial growth factor-alpha, Bax, and Bcl-2 expression. J Cataract Refract Surg. 2009;35(2):354-362.

14. Arnarsson A, Stefánsson E. Laser treatment and the mechanism of edema reduction in branch retinal vein occlusion. Invest Ophthalmol Vis Sci. 2000;41(3):877-879.

15. Stefansson E, Landers MB 3rd, Wolbarsht ML. Increased retinal oxygen supply following pan-retinal photocoagulation and vitrectomy and lensectomy. Trans Am Ophthalmol Soc. 1981;79: 307-334.

16. Han DP, Mieler WF, Burton TC. Submacular fibrosis after photocoagulation for diabetic macular edema. Am J Ophthalmol. 1992;113(5):513-521. 
17. Fong DS, Girach A, Boney A. Visual side effects of successful scatter laser photocoagulation surgery for proliferative diabetic retinopathy: a literature review. Retina. 2007;27(7):816-824.

18. Kozak I, Oster SF, Cortes MA, et al. Clinical evaluation and treatment accuracy in diabetic macular edema using navigated laser photocoagulator NAVILAS. Ophthalmology. 2011;118(6):1119-1124.
19. Al-Hussainy S, Dodson PM, Gibson JM. Pain response and follow-up of patients undergoing panretinal laser photocoagulation with reduced exposure times. Eye (Lond). 2008;22(1):96-99.

20. Roberts CJ, MacLeod JD, Elkington AR. Ocular pain: a casualty study. The spectrum and prevalence of pain in acute eye disease. Eye (Lond). 1997;11(Pt 3):342-344.

\section{Publish your work in this journal}

Clinical Ophthalmology is an international, peer-reviewed journal covering all subspecialties within ophthalmology. Key topics include: Optometry; Visual science; Pharmacology and drug therapy in eye diseases; Basic Sciences; Primary and Secondary eye care; Patient Safety and Quality of Care Improvements. This journal is indexed on

\section{Dovepress}

PubMed Central and CAS, and is the official journal of The Society of Clinical Ophthalmology (SCO). The manuscript management system is completely online and includes a very quick and fair peer-review system, which is all easy to use. Visit http://www.dovepress.com/ testimonials.php to read real quotes from published authors. 